\title{
Novel 2-D Histogram-Based Soft Thresholding for Brain Tumor Detection and Image Compression
}

\author{
Chiranjeevi Karri, Cloud Computing Competence Center (C4), University of Beira Interior, Covilhã, Portugal* \\ G. Ramesh Babu, Raghu Engineering College (Autonomous), Visakhapatnam, India \\ P. M. K. Prasad, GVP College of Engineering for Women, Visakhapatnam, India
}

M. S. R. Naidu, Aditya Institute of Technology and Management, Srikakulam, India

\begin{abstract}
The objective of image compression is to extract meaningful clusters in a given image. Significant groups are possible with absolute threshold values. 1-D histogram-based multilevel thresholding is computationally complex, and reconstructed image visual quality is comparatively low because of equal distribution of energy over the entire histogram plan. So, 2-D histogram-based multilevel thresholding is proposed in this paper by maximizing the Renyi entropy with a novel hybrid genetic algorithm, particle swarm optimization, and symbiotic organisms search (hGAPSO-SOS), and the obtained results are compared with state-of-the-art optimization techniques. Recent study reveals that PSNR fails in measuring the visual quality because of mismatch with the objective mean opinion scores (MOS). So, the authors incorporate a weighted PSNR (WPSNR) and visual PSNR (VPSNR). Experimental results examined on magnetic resonance images of brain and results with 2-D histogram reveal that hGAPSO-SOS method can be efficiently and accurately used in multilevel thresholding problem.
\end{abstract}

\section{KEYWORDS}

2-D Histogram, Genetic Algorithm, Image Compression, Image Thresholding, Particle Swarm Optimization, Ryeni Entropy, Symbiotic Organisms Search

\section{INTRODUCTION}

Image compression is a technique of showing the images in procedural manner, that which reduce the number of bits essential to represent an image and in order to advance the capacity of the storage device. There are several techniques which are proposed by various researchers, but the most used image compression technique is Joint Photographic Expert Group (JPEG). Discrete Cosine Transformed (DCT) was firstly introduced later JPEG-2000 is introduced (Skodras et al., 2001).

There are many methods can be utilized for image compression, some of these methods depend on mathematical transforms such as; discrete cosine transform (Haweel et al., 2016), Discrete Wavelet Transforms (DWT) (Bruylants et al., 2015), Integer Wavelet Transforms (IWT) (Zhang and Tong, 2017), Karhunen Loeve Transforms (KLT) (Zhang \& Tong, 2017), Hartley Transform (Sunder et al.,), Watershed Transform (Hsu, 2012), Walsh Hadamard Transform (WHT) (Venugopal et al., 2016), Tchebichef Transform Kiruba \& Sumathy, and Singular Value Decomposition (SVD) (Kumar \& Vaish, 2017). 
Various clustering algorithms have been proposed for image compression and are categorized into two categories: hierarchical algorithm and partition algorithm (Jain et al., 2000). In Partition cluster, clusters are updated as per the smaller difference between the centroid and input data which is to be cluster. The entire input data is portioned into some clusters based on nearest distance between centroid and data. Whereas in hierarchical cluster, hierarchy (most important) is given to some clusters and are of two types: Agglomerative and Divisive. One can find detailed description of both in (Han et al., 2017).

Image Compression can also be done by non-transformed methods like Vector Quantization (VQ) and Thresholding. In image processing, selecting a gray level threshold from the images like gray level image/color and extracting its background image is a challenging task. Hence many techniques for distinguished gray level threshold are proposed by the researchers. Thresholding is generally applied because of its progressive robustness, accuracy and less time convergence. There are two ways of approaching thresholds, firstly parametric and the other is non- parametric. In nonparametric approach, depending on class variance thresholding it is performed as in Otsu technique or depending on the criterion of entropies like Shannon, Fuzzy and Kapur's (De Luca \& Termini, 1972). If in case, the image is divided into two classes which are object and background, then the threshold is called multi-level threshold or bi-level threshold. Thresholding techniques up hold various real time applications.

A detailed research on image thresholding was performed by Sezgin and Sankur in 2004 and classified it into six categories; those are Clustering-based, Entropy-based, Histogram shape-based, Object attribute-based, spatial and local methods respectively (Sezgin \& Sankur, 2004). Considering the histogram's of the gray level images, Kaur classified the images based on calculating threshold (Kaur et al., 2007). The image is portioned into desired classes based on optimizing the class variance and once example of such kind is Otsu's method (Otsu, 1979). Under bi-level thresholding these two methods found effective in case of more than one threshold whereas in multi-level thresholding the complexity is high. The drawback of Bayesian error and Birge-Massart thresholding is that the computational or CPU time is exponentially rising with the problem and to overcome these problems (Rather \& Bala, 2020), evolutionary and swarm-based calculation techniques are alternatives (Rather $\&$ Bala, 2020). Repositories of the codes are found in citations (Sajad Ahmad Rather, 2020), (Sajad Ahmad Rather, 2020) and (Seyedali Mirjalili, 2020)

Chen-Kuei and Wen-Hsiang in 1998 proposed a principle based on moment preserving, for effective and efficient color image thresholding (Kaur et al., 2007) followed a particular approach for the selecting the wavelet packets with low computational cost that optimize the operational Rate Distortion (R-D), thresholds and quantizers with minimum description length framework to develop JTQ-WP. An inbuilt matlab function was used by (Sidhik, 2015) for the compression i.e Birge-Massart thresholding and the results are compared with unimodal thresholding. Tony and Zhou proposed a technique for noise removal and image compression in wavelet domain thresholding which is based on Partial Differential Equation (PDE) and it has the advantage of variations in framework (Chan \& Zhou, 2007). Image is compressed with the help of Multistage Lattice Vector Quantization (MLVQ) and DWT coefficients are thresholded. Proposed method always tries to reduce the quantization error and its computational time is less as compared to normal VQ (Salleh \& Soraghan, 2007). Kaveh et al., proposed image thresholding in frequency domain by transforming the image to be compressed into frequency domain by 2-D discrete wavelet transform. The thresholds are optimized with recently proposed soft computing techniques such as Particle Swarm Optimization (PSO) (Ahmadi et al., 2015). The authors did decomposition of DWT coefficients at three level and among all chosen thresholds, $62.5 \%$ of thresholds are assigned for approximation coefficients and reset thresholds $37.5 \%$ are distributed to diagonal, vertical and horizontal coefficients. The obtained results are compared with mostly used Set Partition in Hirechal Tree (SPHIT), JPEG, Chrysafis and JPEG-2000 and results are proved better in all measuring parameters. The PSO suffers in stability if any particle velocity is very 
high and requires much tuning parameters to tune and care must be taken while selecting the tuning parameters (Chiranjeevi \& Jena, 2018).

In this paper, we applied Hybrid Genetic Algorithm, Particle Swarm Optimization, and Symbiotic Organisms Search for effective and efficient thresholding with the help of 2-D histogram and assumed Renyi entropy as a fitness function which is optimized. The obtained results are compared with 1-D histogram and other state of art optimization techniques. The compressed image is further compressed by encoding techniques such as Runlegth coding followed by Arithmetic coding. For the better performance evaluation of proposed 2-D histogram based image thresholding we consider objective function value, peak signal to noise ratio (PSNR), weighted peak signal to noise ratio (WPSNR) and Visual PSNR (VPSNR). The performance of 2-D histogram based image thresholding is superior in terms of these parameters compared to other algorithms

\section{CONCEPT OF RENYI ENTROPY}

Let's assume an 'n' array finite discrete Probability Distributions (pdf) such as $\left(F_{1}, F_{2}, F_{3} \ldots F_{n}\right) \varepsilon \Delta_{n}$ where $\Delta_{n}=\left\{\left(F_{1}, F_{2}, F_{3} \ldots F_{n}\right), F_{i} \geq 0, i=1,2,3 \ldots . . n, n \geq 2, \sum_{i=1}^{n} F_{i}=1\right.$. with random variables $\left(X_{l}\right.$, $X_{2}, X_{3}, \ldots \ldots X_{n}$ ) then Renyi entropy for independent and additive random events is given as (Rényi, 1961).

$$
H_{\alpha}=\frac{1}{1-\alpha} \log _{2}\left(\sum_{i}^{n} F_{i}^{\alpha}\right)
$$

Where ' $\alpha$ ' is greater than zero and it is called as entropy order. When ' $\alpha$ ' tends to one then Renyi entropy becomes Shannon entropy. In general image is clustered in to two clusters, one carries object information (cluster $C_{1}$ ) and another carries background (cluster $C_{2}$ ) then Renyi entropy is

$$
\begin{aligned}
& H_{\alpha}\left[C_{1}\right]=\frac{1}{1-\alpha}\left[\log _{2}\left(\sum_{i=0}^{t}\left(\frac{F(i)}{F\left(C_{1}\right)}\right)^{\alpha}\right)\right] . \\
& H_{\alpha}\left[C_{2}\right]=\frac{1}{1-\alpha}\left[\log _{2}\left(\sum_{i=t}^{L-1}\left(\frac{F(i)}{F\left(C_{2}\right)}\right)^{\alpha}\right)\right] .
\end{aligned}
$$

Where $F\left(C_{1}\right)=\sum_{i=0}^{t} F(i) . F\left(C_{2}\right)=\sum_{i=t+1}^{L-1} F(i)$. Here $F_{i}$ is the normalized one dimensional histogram of the image and ' $L$ ' is highest intensity level of gray scale image. The overall Renyi entropy for a given image with one threshold ' $t$ ' is given as

$$
\varphi_{\alpha}(t)=\operatorname{argmax}\left(\left[H_{\alpha}\left[C_{1}\right]+H_{\alpha}\left[C_{2}\right]\right]\right) .
$$




\section{Multi-Level Thresholding}

Let image is divided into ' $N$ ' number of clusters $C=\left(C_{1}, C_{2}, C_{3} \ldots C_{N}\right)$ with $N$ number of threshold values $t=\left(t_{1}, t_{2}, t_{3} \ldots t_{N}\right)$ then Renyi entropy for each individual cluster is defined as [22]

$$
H_{\alpha}\left[C_{1}\right]=\frac{1}{1-\alpha}\left[\log _{2}\left(\sum_{i=0}^{t_{1}}\left(\frac{F(i)}{F\left(C_{1}\right)}\right)^{\alpha}\right)\right] .
$$

$H_{\alpha}\left[C_{2}\right]=\frac{1}{1-\alpha}\left[\log _{2}\left(\sum_{i=t_{1}+1}^{t_{2}}\left(\frac{F(i)}{F\left(C_{2}\right)}\right)^{\alpha}\right)\right]$

$$
H_{\alpha}\left[C_{N}\right]=\frac{1}{1-\alpha}\left[\log _{2}\left(\sum_{i=t_{N}-1}^{L-1}\left(\frac{F(i)}{F\left(C_{N}\right)}\right)^{\alpha}\right)\right]
$$

Where $F\left(C_{1}\right)=\sum_{i=0}^{t_{1}} F(i) \cdot F\left(C_{2}\right)=\sum_{i=t_{1}+1}^{t_{2}} F(i) \cdot F\left(C_{N}\right)=\sum_{i=t_{N}-1}^{L-1} F(i)$. the overall Renyi entropy or objective function for a given image for $N$ thresholds is given as

$$
\varphi_{\alpha}(t)=\operatorname{argmax}\left(\left[H_{\alpha}\left[C_{1}\right]+H_{\alpha}\left[C_{2}\right]+\ldots \ldots \ldots \ldots H_{\alpha}\left[C_{N}\right]\right]\right) .
$$

For simplifying the calculations, two dummy thresholds are introduced $t_{0}$ and $t_{N}=L-1$ which satisfy the condition $t_{0}<t_{1 \ldots \ldots \ldots}<t_{N-1}<t_{N}$. The optimal thresholds are obtained by maximizing the above equation with any soft computing technique.

\section{Two-Dimensional Renyi Entropy}

Let $\mathrm{I}(m, n)$ is an image intensity at spatial location $(m, n)$. In digital image $[I(m, n) \mid m \varepsilon\{1,2,3, \ldots \ldots$, $M\}, n \varepsilon\{1,2,3, \ldots \ldots ., N\}$, where ' $M$ ' and ' $N$ ' are size of the image and its 1D-histogram $h(x)$ for $x$ $\varepsilon\{1,2,3, \ldots \ldots, L-1\}$, where ' $L$ ' is 256 for gray scale image. Let denote elements in histogram $\{1$, $2,3 \ldots 255\}$ as $G$. In literature, optimal thresholds selection is based on 1D-histogram are obtained by optimizing the objective function/entropy.

The 2-D histogram of an image is obtained by defining a local average of pixel, $I(x, y)$, as the average intensity of its nine neighbors denoted as $g(x, y)$ (Sarkar \& Das, 2013)

$$
g(x, y)=\frac{1}{9} \sum_{i=-1}^{1} \sum_{j=-1}^{1} f(x+i, y+j)
$$


For example let us take an image of size $4 * 4$ as shown in figure 1 (a) and its average intensity $g(x, y)$ is calculated by padding required number of zeros at edges as shown in figure 1 (b). First table in figure is image and first element i.e 126, $g(x, y)$ is calculated by padding zero's at edges as in figure and last tables shows $g(x, y)$ for entire image $I(x, y)$. 2-D histogram of Metastatic image at marked area is shown in figure 2 , where diagonal quadrants carry much information.

Figure 1. Example for 2-D histogram calculation

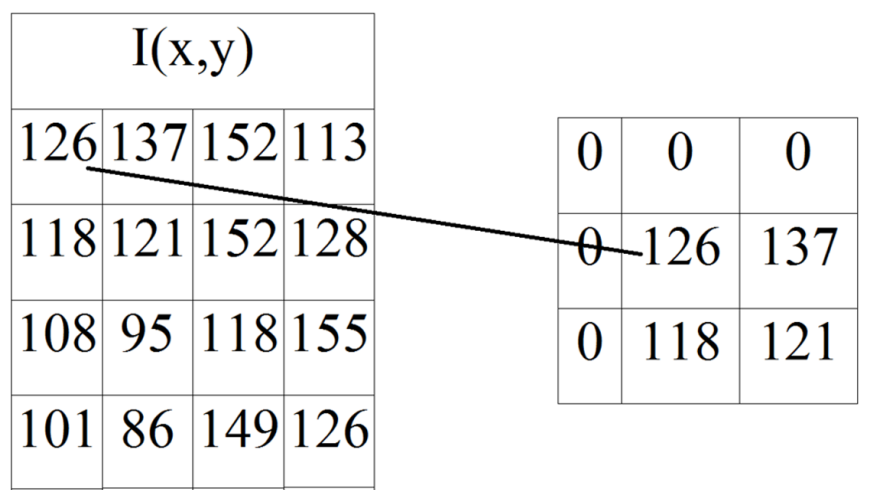

\begin{tabular}{|c|c|c|c|}
\hline \multicolumn{4}{|c|}{$g(x, y)$} \\
\hline 56 & 90 & 89 & 61 \\
\hline 78 & 125 & 130 & 91 \\
\hline 70 & 116 & 126 & 92 \\
\hline 43 & 73 & 81 & 61 \\
\hline
\end{tabular}

The 2-D histogram of tested images as in figure 2 and are divided into four clusters by a single threshold $(t, s)$. Where $t$ is threshold for original image intensity $I(x, y)$ and $s$ is threshold for average intensity image $g(x, y)$. The divided cluster area is not same. The first diagonal quadrants represents object, third represent background, second and fourth quadrants are neglected because of these does not carry any information (pair occurrence is less) as show in figure 2. The Renyi entropy for object and background is given as

Figure 2. Metastatic image and 2-D histogram

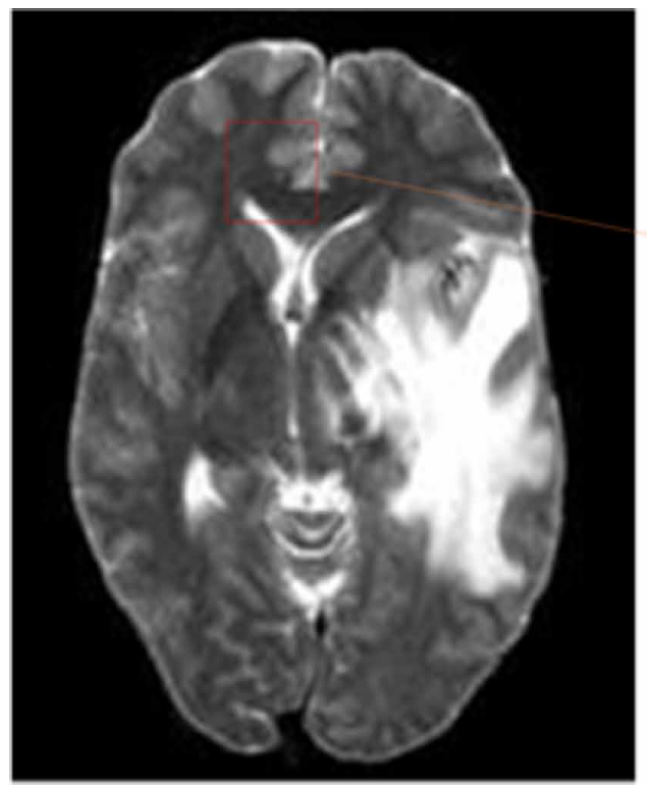

\begin{tabular}{|c|c|c|c|}
\hline \multicolumn{4}{|c|}{ 2-D histogram } \\
\hline 21 & 1 & 1 & 0 \\
\hline 5 & 19 & 2 & 1 \\
\hline 8 & 7 & 24 & 4 \\
\hline 4 & 6 & 7 & 16 \\
\hline
\end{tabular}




$$
H_{\text {object }}^{\alpha}[t, s]=\frac{1}{1-\alpha}\left[\log _{2} \sum_{i=0}^{t}\left(\sum_{j=0}^{s}\left(\frac{F(i, j)}{F_{D 1}(t, s)}\right)^{\alpha}\right)\right]
$$

$H_{\text {background }}^{\alpha}[t, s]=\frac{1}{1-\alpha}\left[\log _{2} \sum_{i=t+1}^{L-1}\left(\sum_{j=s+1}^{L-1}\left(\frac{F(i, j)}{F_{D 2}(t, s)}\right)^{\alpha}\right)\right]$

Where $F_{D 1}(t, s)=1-\sum_{i=0}^{t} \sum_{j=0}^{s} F(i, j)$ and $F_{D 2}(t, s)=1-\sum_{i=t+1}^{L-1} \sum_{j=s+1}^{L-1} F(i, j)$

The final objective function which is to maximized for better threshold $(t, s)$ selection is

$$
\varphi_{\alpha}(t)=\operatorname{argmax}\left(\left[H_{\text {object }}^{\alpha}[t, s]+H_{\text {background }}^{\alpha}[t, s]\right]\right)
$$

\section{Proposed Renyi 2D-Hisotgram Based Multi-Level Thresholding}

Multilevel thresholding with 1-D histogram deliver inferior results because of incorrect selection of thresholds, so recent study proved that thresholding with 2-D histogram deliver superior results especially in multilevel thresholding. Multilevel thresholding gained lots of popularity over bi- level thresholding because; it clusters the image into several useful clusters, helps for accurate analysis and interpretation of the image. In this paper, we proposed 2-D Renyi entropy based multilevel thresholding for image compression by incorporating the advantage of 2-D histogram. If the 2-D histogram of an image is clustered into 9 clusters with two thresholds $\left(t_{1}, t_{2}\right)$ and $\left(s_{1}, s_{2}\right)$ as shown in figure 3 (a). Then the diagonal quadrants $1^{\text {st }}, 5^{\text {th }}$ and $9^{\text {th }}$ represents objects, intermediate regions and background respectively as illustrated in Figure 3 (a) and reset of the regions are noise edges which are ignored. The Renyi entropy of diagonal quadrants are given as

$$
\begin{aligned}
& H_{\text {object }}^{\alpha}[t, s]=\frac{1}{1-\alpha}\left[\log _{2} \sum_{i=0}^{t_{1}}\left(\sum_{j=0}^{s_{1}}\left(\frac{F(i, j)}{F_{D 1}(t, s)}\right)^{\alpha}\right)\right] \\
& H_{\text {intermediate }}^{\alpha}[t, s]=\frac{1}{1-\alpha}\left[\log _{2} \sum_{t_{1}+1}^{t_{2}}\left(\sum_{s_{1}+1}^{s_{2}}\left(\frac{F(i, j)}{F_{D 2}(t, s)}\right)^{\alpha}\right)\right]
\end{aligned}
$$


Figure 3. 2-D histogram for a) 3- level b) 4- level

\begin{tabular}{|c|c|c|c|c|}
\hline \multicolumn{2}{|c|}{$(0,0)$} & \multicolumn{3}{|c|}{$\mathrm{t}_{2} \quad(0, \mathrm{~L}-1)$} \\
\hline & & 2 & 3 & \\
\hline $\mathbf{s}_{1}$ & 4 & 5 & 6 & \\
\hline $\mathrm{S}_{2}$ & 7 & 8 & 9 & \\
\hline (L & & & (L-1, & $-1)$ \\
\hline
\end{tabular}

(a)

\begin{tabular}{|c|c|c|c|c|c|}
\hline \multicolumn{2}{|c}{$(0,0)$} & $\mathrm{t}_{1}$ & $\mathrm{t}_{2}$ & $\mathrm{t}_{3}$ & $(0, \mathrm{~L}-1)$ \\
\hline & 1 & 2 & 3 & 4 & \\
$\mathrm{~s}_{1}$ & & & & & \\
\cline { 2 - 6 } $\mathrm{s}_{2}$ & 5 & 6 & 7 & 8 & \\
\cline { 2 - 6 } $\mathrm{s}_{3}$ & 9 & 10 & 11 & 12 & \multicolumn{5}{|c|}{$(\mathrm{L}-1, \mathrm{~L}-1)$} \\
\cline { 2 - 6 } & 13 & 14 & 15 & 16 \\
\hline \multicolumn{2}{|c|}{$(\mathrm{L}-1,0)$} \\
\end{tabular}

(b)

Figure 4. Input images and corresponding 2-D histogram
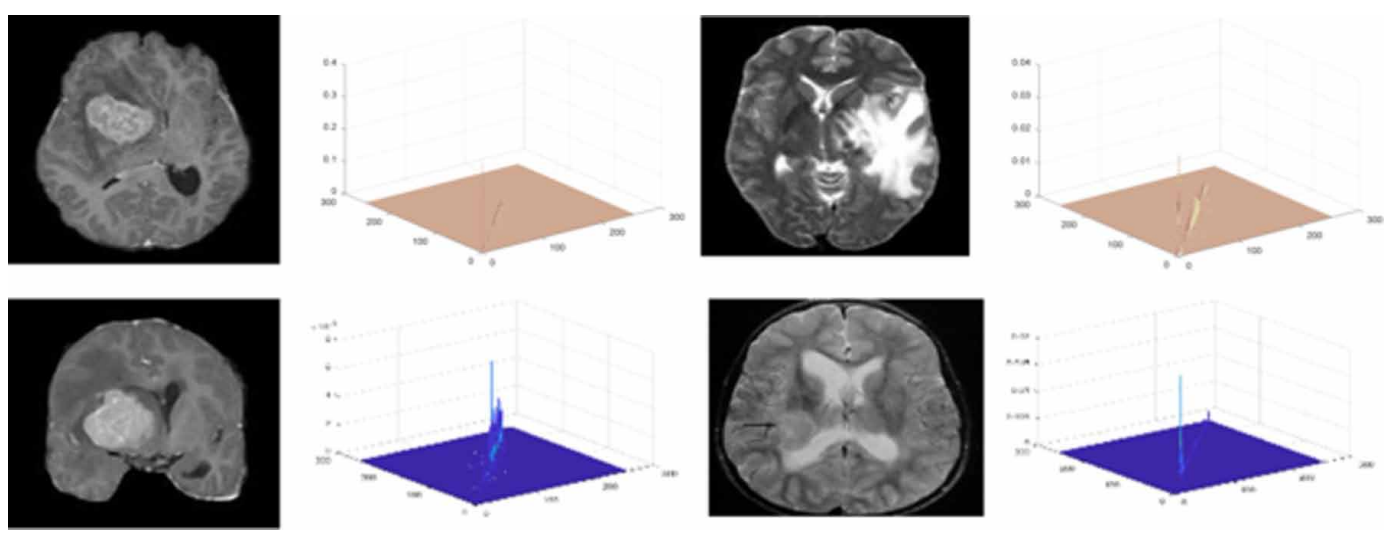

$H_{\text {backgroud }}^{\alpha}[t, s]=\frac{1}{1-\alpha}\left[\log _{2} \sum_{t_{2}+1}^{L-1}\left(\sum_{s_{2}+1}^{L-1}\left(\frac{F(i, j)}{F_{D 3}(t, s)}\right)^{\alpha}\right)\right]$

Where $F_{D 1}(t, s)=1-\sum_{i=0}^{t_{1}} \sum_{j=0}^{s_{1}} F(i, j), F_{D 2}(t, s)=1-\sum_{i=t_{1+1}}^{t_{2}} \sum_{j=s_{1}+1}^{s_{2}} F(i, j)$ and $F_{D 3}(t, s)=1-\sum_{i=t_{2+1}}^{L-1} \sum_{j=s_{2}+1}^{L-1} F(i, j)$

The final objective function which is to maximized for better threshold $(t, s)$ selection is 
$\varphi_{\alpha}(t)=\operatorname{argmax}\left(\left[H_{\text {object }}^{\alpha}[t, s]+H_{\text {intermediate }}^{\alpha}[t, s]+H_{\text {background }}^{\alpha}[t, s]\right]\right)$

Above equation can be extended for ' $N$ ' threshold values as given below

$\varphi_{\alpha}(t)=\operatorname{argmax}\left(\left[H_{1}^{\alpha}[t, s]+H_{2}^{\alpha}[t, s]+H_{3}^{\alpha}[t, s] \ldots \ldots \ldots \ldots H_{N+1}^{\alpha}[t, s]\right)\right.$

Where $H_{k}^{\alpha}[t, s]=\frac{1}{1-\alpha}\left[\log _{2} \sum_{i=t_{k-1}+1}^{t_{k}}\left(\sum_{j=s_{k-1}+1}^{s_{k}}\left(\frac{F(i, j)}{F_{D k}(t, s)}\right)^{\alpha}\right)\right]$

For simplifying the calculations, two dummy thresholds are introduced $t_{0}$ and $t_{N}+1=L-1$ which satisfy the condition $t_{0}<t_{1} \ldots \ldots \ldots t_{N-1}<t_{N}+1$. Similarly two dummy variable are introduced $s_{0}$ and $s_{N}+1=L-1$ which satisfy the condition $s_{0}<s_{1 \ldots \ldots \ldots .}<s_{N-1}<s_{N}+1$. The 2-D histogram of four standard images are shown in figure 4 and form these figure it is observed that most of the information/energy is concentrated on diagonal quadrants. Multilevel thresholding is a time consuming process and is proportional to the number of thresholds ' $N$ '. So, soft computing techniques play a significant role in this contest by assuming Eq. (17) as an objective function. This leads to reduction in the computational time. Figure. 4 shows input images and respective 2-D histogram.

\section{OVERVIEW OF HYBRID GA-PSO-SOS (HGAPSO-SOS)}

- Genetic Algorithms (GA): It was initiated and developed during 1960 to 1970 by team Holland team and had been using to solve many constrained and unconstrained optimization problems. It is inspired and developed by in depth study of natural selection of Charles Darwin's theory (Yang, 2010). It is a non-swarm based optimization technique for optimization of any problem. It was developed by observing the human intelligence cause for the difference in intelligence. It is proved that GA can solve any problem which may be any four combinations between continuous, linear, discrete and non-linear. GA being a non-swarm based technique consists of chromosome for each and every population or solution of the problem. These chromosomes may be represented in two ways. First one is binary representation, where all the solutions and operations are in the form of binary digits and second one is floating point, where all functions are in floating point numbers. Floating points includes real numbers where all the real numbers and dimensions of the problems are equal to the number of variables which are to be optimized. Initial population are generated by a random numbers with in the range of search space and are depends on the maximum and minimum value of parameters to be optimized. Now select a best solution based on the fitness function and generate a new population with any of the four selection methods those are fitness proportional, ranked based, tournament and Roulette wheel selection. The ordinary GA uses two steps for selection and creation of new population i.e mutation operation and crossover operation. The newly generated population or chromosomes are named as offspring (Grosan \& Abraham, 2011). The crossover operation is performed between two parents for generation of new healthy child. The crossover operation is performed based on two assumptions:

- All the operations are in discrete form and all obey the binary operation rules.

- Assigning new real values using operators for offspring position that lies between parent values. They are called as intermediate or arithmetic recombination. Let $A$ and $B$ are the parents then offspring $C$ is computed with the following equation 
$C_{i}=\alpha \cdot A_{i}+(1-\alpha) \cdot B_{i} \alpha \in[0,1]$

In all iterations chromosomes changes its values by mutation operation. This operation is applied on chromosome parent for generation of new offspring. Like crossover, mutation also has some variants based on chromosome. Mutation in real number is obtained by addition of chromosome with randomly created real number or randomly generated number from Gaussian (normal) distribution. Let $A$ is chromosome and its $i^{\text {th }}$ variable is $A_{i}$ then new offspring $A^{l}$ is obtained by mutating $i^{t h}$ gene $A_{i}^{l}$ and is calculated with the following equation:

$A_{i}^{l}=A_{i}+N, N=\operatorname{rand}(0,1) .[U B-L B / M], L B<A_{i}$ and $A i^{l}<U B$

Where ' $N$ ' is random number or value taken form Gaussian distribution and ' $M$ ' is random real number (range between 1 and 1000 (mostly authors prefer $M$ value 10). rand $(0,1)$ is a random number lies between 0 to $1 . L B$ and $U B$ are upper limit and lower limit of $A_{i}$ and $A_{i}^{l}$. The genetic algorithm is explained in algorithm 1.

Algorithm 1: Genetic algorithm

Step 1: Initialize number of chromosome as $N$.

Step 2: Calculate the objective function/fitness function for all

$N$ chromosome.

Step 3: N chromosome are updated by four repeated steps i.e best

chromosome selection -

Crossover operation and Mutation operation and

finally Replacement

Step 4: The generated chromosome are forwarded for next iterations

Step 5: Repeat step 2 - 4 till stopping criteria or maximum

iteration

Particle swarm optimization: It is inspired by the searching behavior of particles; some examples are swarm of fish or birds and were developed in the year 1995 by Eberhart and Kennedy [25]. Unlike GA, the PSO does not go throw crossover and mutation instead it follows randomness and some intelligence in updating of the both particle positions and velocity. The PSO is simple and easily adopted for any particle and mathematical problems. While searching for answer to any problem it follows a specific strategy. Each particle in PSO may be assumed as one bird or one fish and are indicated with $O_{i}$. All particles are always tried to become a best among and change their positions with a specific strategy. In beginning, some number of populations are initialized which search entire search space. Each particle gain some initial velocity $V_{i}$ and position $Q_{i}$ of dimensions equal to dimensions of the problem. In all iterations each particle hold some position called personal best $\left(O_{p}\right)$ and highest fitness particle holds global best $\left(O_{b e s}\right)$ position and these positions are updated in upcoming iterations (Karri \& Jena, 2016).

Algorithm 2: PSO

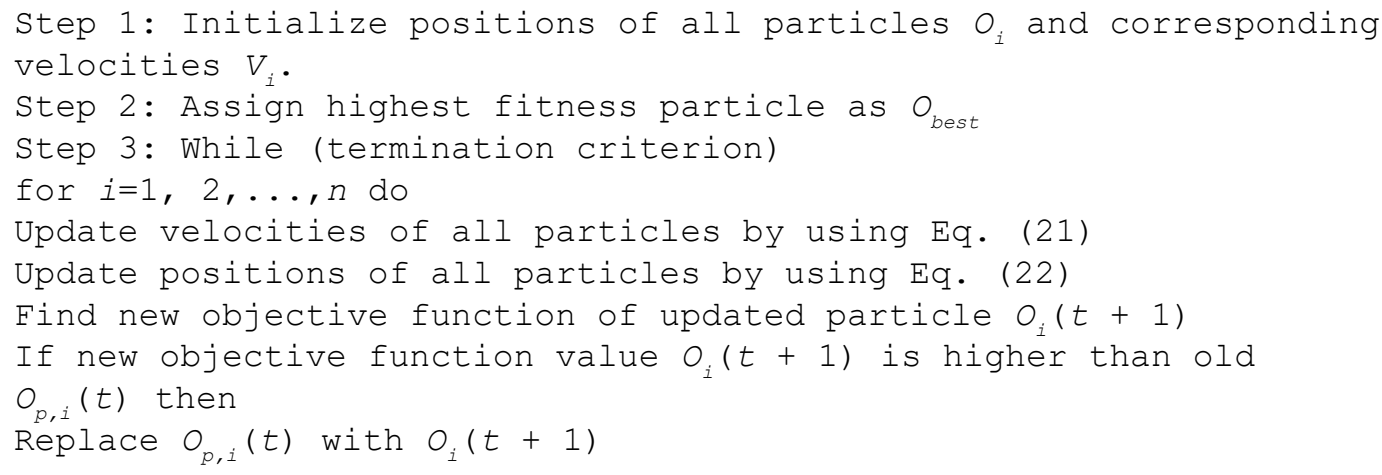




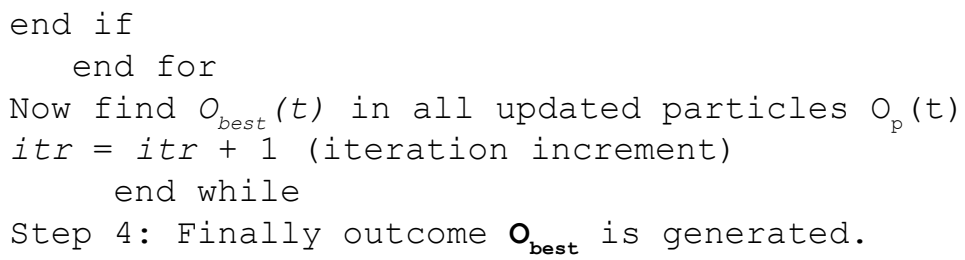

Let ' $t$ ' is current iteration, then PSO velocity and position update follows Eq. 21 and Eq. 22

$$
\begin{gathered}
V_{i, d}(t+1)=V_{i, d}(t)+C_{1 *} r_{1}\left(O_{p(i, d)}(t)-O_{i, d}(t)\right)+ \\
\left.c_{2 *} r_{2}(O) \operatorname{best(d)}(t)-O_{i, d}(t)\right) \\
O_{i, d}(t+1)=O_{i, d}(t)+V_{i, d}(t+1)
\end{gathered}
$$

Eq. (21) is for velocity updation and Eq. (22) is for updation of particle position with the help of updated velocities. $O_{p(i, d)}$ is a personal best for the $d^{\text {th }}$ dimension of particle $I$ and $O_{\text {best }}$ is the best particle among all particle in current iteration ' $t$ ', $c_{1}$ and $c_{2}$ are user defined control tuning parameters, $r_{1}$ and $r_{2}$ are random numbers lies between 0 to 1. The PSO algorithm is given in Algorithm 2.

Symbiotic Organisms Search (SOS): SOS is a soft computing techniques developed based on organisms and was proposed in the year 2014 by Prayogo and Cheng, it is inspired from symbiotic organisms that used to survive in the ecosystem (Cheng \& Prayogo, 2014). SOS is developed for continuous search space. The projected SOS algorithm tries to pursue the fitness organism and simulates symbiotic interactive behavior amongst the paired organism relationship in the ecosystem. The individuals in the population are known as organism and every organism signifies a point in the search space, hence obtaining the possible optimal global solution is achieved to the problem. The fitness for every organism shows the degree of adaption to the treated goal. This algorithm has various advantages over the other algorithms: The major advantage of SOS is that, it does not require prior tuning of tuning parameters. As like other algorithms, SOS updates all organism position in ever iteration. Position update is done in three successive operations; those are mutualism, commensalism and parasitism. The organism positions changed based on best possible relation among all. The first step mutualism gives a benefit to both organisms as in bees and flowers. The second commensalism gives some value to organism by making a relation between fish and shark. Third one parasitism gives benefit to shark without the effect the relation with fish. In all phases, the positions are updated if and if relation leads to best objective value for $i$ or $j$ organisms. The above discussion is summarized in algorithm 3 . In every iteration, update the all phases with the corresponding equations and are as follows.

Algorithm 3: SOS

1. Initialize the required parameters

2. While (until stopping criterion) do

Three phases 1. Mutualism 2. Commensalism 3. Parasitism

3. End while

Mutualism phase: In this phase, both the organisms are benefited related to the relationship between flowers and bees. In this stage, organism $O_{j}$ randomly selected and is interact with the other organism $O_{i}$. They maintain a good relationship between them so that both organisms got benefited. The updated positions of both the organisms are obtained with the following equations.

$$
\begin{gathered}
O_{\text {inew }}=O_{i}+\operatorname{rand}(0,1) \cdot\left(O_{\text {best }}-\text { Mutual_vector. } B F_{1}\right) \\
O_{\text {jnew }}=O_{j}+\operatorname{rand}(0,1) \cdot\left(O_{\text {best }}-\text { Mutual_vector. } B F_{2}\right)
\end{gathered}
$$


Where mutal_vector gives relationship between the organisms $O_{i}$ and $O_{j}$ and above equation explains the efforts of mutualistic in gaining their goals and enhance their living survival. The benefit factors $B F_{1}$ and $B F_{2}$ shows how much of benefit organism acquired while interacting with other organism. These two are randomly selected must be either 1 or $2 . O_{\text {best }}$ is the best degree of adaption that has founded until now.

Commensalism phase: This phase is developed on the basis of relation between the Remora fish and sharks. The remora always receives benefits whereas shark may or may not receive benefits from relationship. As disused in mutualism phase, in this randomly selected $O_{j}$ organism gets benefit by maintaining a relationship with other organism $O_{i}$. Then updated equation is (26)

$O_{\text {inew }}=O_{i}+\operatorname{rand}(-1,1) \cdot\left(O_{\text {best }}-O_{j}\right)$

Parasitism phase: This phase exit between the human being and malaria mosquito, in which human being got effected and some time may die and mosquito got benefited with the relationship. As one of the organisms got affected, so need to replace with newly generated organism. As like other phases, one organism is selected randomly $O_{j}$ and it acts a victim for parasite vector. In problem search space, this vector is obtained by replacing $O_{j}$ with newly generated and then modify the randomly chosen organism. If at all this vector is better as compared to $O_{j}$ then this phase kill $O_{j}$ and replace or else $O_{j}$ gains some energy from parasite and live for some other days.

Hybrid algorithm based on GA, PSO and SOS:

Developed by Charles Darwin for the first time by merging of three of the evolutionary algorithms GA, PSO and SOS represented as hGAPSO-SOS, has been taken from natural selection (Yang, 2010). As per authors view, organisms always try to become strong in life by competent with other organism so all organisms have better probability to propagate and leads to a better offspring. These offspring will receive the features which finished the parents ending well. These features of organisms will spread to all population in search space and this process is called evolution (Gregory, 2009). Here is fact that if any organism has good genetic structure leads a better feature, henceforth better and has long life in ecosystem. This kind of behavior leads to better relation with others organism. Each organism increase life span and their experiences will be shared to children's for better life. In the proposed algorithm we took the advantage of incorporating the SOS with GA. The GA creates a better offspring with good genetic structure from parents. The PSO algorithm gives some important experiences to all organisms which leads better survival of each organism. In the proposed algorithm GA follows PSO and combination follows SOS.

In all iterations, the hGAPSO-SOS starts with GA with required population, dimension of problem and required initialization of parameters. The selection operation of GA is useful for selection of best population which is obtained after doing some required or mandate operations, that is mutation followed by crossover operation. The global best solution $O_{b e s t}$ is also selected among all populations. The outcome of GA populations is obtained by incorporating parent's experiences and corresponding offspring. There are four parameters in each offspring those are position, velocity, best experience (which itself consists of two fields position and cost) and cost. In the next step all organisms get some best experience with PSO. If any organism position is better as compared to past position then it will move to better level or else it will remain in same position. If best experience is better than the global best $O_{\text {best }}$ then replace it with new position (Chiranjeevi et al., 2017). So PSO always try to check for better position by updating the velocity and keep best for the next iterations and parallel it update the $O_{\text {best }}$. As all the organisms got some experience with the PSO, now they try to establish 
a better relation with other organism which leads to better offspring and healthy population. In third phase, if any organism got improved fitness value then that organism position is updated with SOS interaction if not nothing do. From whole observation the GA and SOS are useful for position update PSO update the $O_{\text {best }}$ and personal best of organism. If $O_{\text {best }}$ value in current iteration is equal to stopping condition, then algorithm stops or else same process is repeated.

\section{RESULTS AND DISCUSSION}

The proposed algorithm performance is evaluated by considering the standard benchmark images like "Astrocytoma", "Coronary", "Glioma", and "Meningioma" and all the images are of size 256×256 each pixels takes 8 bits (bits per pixel $=8$ ). These images are captured from Siemens-Area MRI scanner equipment, in which image is captured on slice of thickness $1 \mathrm{~mm} \times 1 \mathrm{~mm} \times 1 \mathrm{~mm}$ by 48 multi channels with 1.5 Tesla magnetic field intensity. All the images are in '.tif' format except Meningioma which is in .gif and Coronary is in .jpeg format. All the algorithms are simulated in Matlab version 2017 and implemented in desktop with specifications: Windows 7 Enterprise N, HP Compaq LE1902x, Intel (R) Core (TM) Duo CPU e7500N at 2.93GHz, 64-bit operating system. The number of iterations itr $=50$, population size $P=100$, upper bound $U B=255, L B=0$, dimensions of the problem $D=t h$ are considered for all optimization algorithms. In this paper, number of thresholds $(t h=5)$ selected for all algorithms because the number of thresholds in published previous paper is 5 . In this paper, performance of the proposed algorithm is tested by thresholding the image with the help of both 1-D histogram and 2-D histogram compared the results with the PSO, BFOA and hBFOA-PSO. The same tuning parameters as in (Chiranjeevi \& Jena, 2017) and (Naidu et al., 2018) are taken for PSO, BFOA.

\section{Quantitative Validation}

To inspect the influence of the hGAPSO-SOS algorithm for the problem of multilevel thresholding, we considered Renyi entropy as objective function or fitness function. The Hybrid Genetic Algorithm Particle Swarm Optimization Symbiotic Organisms Search and other three algorithms are applied on Renyi entropy objective function. Also, the results of the hGAPSO-SOS are compared with PSO, BFOA and hBFOA-PSO in both 1-D and 2-D histogram. To maximize the objective function all the algorithms are optimized. Table 1 show the objective function for hGAPSO-SOS and are compared with PSO, BFOA and hBFOA-PSO. Hence, from Table. 1, by using Renyi entropy the objective value obtained with 2-D histogram for different images are higher than with 1-D histogram and the proposed hGAPSO-SOS are compared with objective value is higher than PSO, BFOA and hBFOAPSO with both histograms. Table 1 also shows the standard deviation of all algorithms. Figure 7 shows the graphical view of objective value of various algorithms.

\section{Mean Square Error (MSE) and Peak Signal to Noise Ratio (PSNR)}

The PSNR illustrates the variations among the decompressed image and input image. In general, it indicates the measure of visual difference of two images and units are decibels $(\mathrm{dB})$. If the reconstructed image shows the better quality, it indicates the higher value of PSNR.

The below equation (27) is to calculate peak signal to noise ratio value, $Y$ is output image and $X$ is input image shown below.

$$
P S N R=10 * \log _{10}\left(\frac{\left(255^{2}\right)}{M S E}\right)
$$

The below equation (28) to calculate mean square error value, $Y$ is output image and $X$ is input image and $M \times N$ is the size of image shown below. 
Table 1. Comparison of Objective Value \& Standard deviation for various algorithms

\begin{tabular}{|l|l|l|l|l|l|}
\hline \multirow{4}{*}{ Images } & \multirow{2}{*}{ Opt Tech } & \multicolumn{2}{c|}{ Objective value } & \multicolumn{2}{c|}{ Standard deviation } \\
\cline { 3 - 6 } & & \multicolumn{1}{|c|}{ 1-D histogram } & \multicolumn{1}{c|}{ 2-D histogram } & \multicolumn{1}{c|}{ 1-D histogram } & \multicolumn{1}{c|}{ 2-D histogram } \\
\hline \multirow{5}{*}{ Meningioma } & BFOA & 16.913 & 17.2727 & 0.410305 & 0.547852 \\
\cline { 2 - 6 } & hBFOA-PSO & 16.922 & 17.2311 & $1.45 \mathrm{E}-14$ & $1.14 \mathrm{E}-15$ \\
\cline { 2 - 6 } & hGAPSO-SOS & 17.745 & 17.3589 & $3.61 \mathrm{E}-15$ & $2.01 \mathrm{E}-15$ \\
\hline \multirow{5}{*}{ Glioma } & PSO & 19.658 & 18.4089 & 0.241575 & 0.125874 \\
\cline { 2 - 6 } & BFOA & 19.824 & 19.9687 & 0.204440 & 0.478956 \\
\cline { 2 - 6 } & hBFOA-PSO & 19.835 & 20.3785 & $3.61 \mathrm{E}-15$ & $1.02 \mathrm{E}-14$ \\
\cline { 2 - 6 } & hGAPSO-SOS & 19.947 & 20.3738 & $3.61 \mathrm{E}-15$ & $2.01 \mathrm{E}-15$ \\
\hline \multirow{5}{*}{ Coronary } & PSO & 18.268 & 20.4214 & 0.369852 & 0.587945 \\
\cline { 2 - 6 } & BFOA & 18.273 & 18.4124 & 0.341585 & 0.014785 \\
\cline { 2 - 6 } & hBFOA-PSO & 18.348 & 18.5245 & $7.23 \mathrm{E}-15$ & $5.01 \mathrm{E}-14$ \\
\cline { 2 - 6 } & hGAPSO-SOS & 18.410 & 18.7458 & $3.61 \mathrm{E}-15$ & $1.45 \mathrm{E}-14$ \\
\hline
\end{tabular}

$\mathrm{MSE}=\frac{1}{\mathrm{M} \mathrm{X} \mathrm{M}} \sum_{\mathrm{i}=1}^{\mathrm{M}} \sum_{\mathrm{j}=1}^{\mathrm{M}}\left(\mathrm{X}_{\mathrm{ij}}-\mathrm{Y}_{\mathrm{ij}}\right)^{2}$

The PSNR values attained from the different algorithms are shown in Table 2, when compared to PSO, BFOA and hBFOA-PSO, the proposed algorithm attains higher PSNR value with 2-D histogram as compared to 1-D histogram. So the quality of the reconstructed images gets much better for the higher level of thresholds. From Table 2, it is also observed that, the 2-D histogram gives higher PSNR value than 1-D histogram and it displays the improved reconstructed image quality with 2-D histogram. The mean square error between original image and reconstructed image is less for hGPSO-SOS compared to other methods as tabulated in Table 2.

The major advantage of PSNR, its simplicity in the calculation while major disadvantage is that it does not consider any of the Human Visual System (HVS) attributes. From figure 5, it is observed that PSNR of Astrocytoma is $77.5538 \mathrm{~dB}$ but visual quality in reconstructed image is very poor whereas in right side Astrocytoma image PSNR value is $30.3473 \mathrm{~dB}$ and in proportion visual quality is also good. In similar for Glioma, PSNR value is $75.2909 \mathrm{~dB}$ but visual quality is not good. From the observation a higher PSNR values may not give good visual quality in reconstructed image. In this case, PSNR value won't be considered as visual quality measure. So there is a need of another other parameters which gives value addition to both quantitative value and visual quality. So in this paper we consider Weighted PSNR (WPSNR) and Visual-PSNR (VPSNR) for accurate performance measure of proposed techniques. 
International Journal of Applied Metaheuristic Computing

Volume $13 \cdot$ Issue 1

Table 2. Comparison of PSNR \& MSE values for various algorithms

\begin{tabular}{|l|l|l|l|l|l|}
\hline \multirow{4}{*}{ Images } & \multicolumn{2}{|l}{ PSNR } & MSE \\
\hline \multirow{5}{*}{ Meningioma } & Opt Tech & 1-D histogram & 2-D histogram & 1-D histogram & 2-D histogram \\
\cline { 2 - 6 } & PSO & 36.84133 & 36.88658 & 13.45697 & 13.31749 \\
\cline { 2 - 6 } & hBFOA-PSO & 38.65026 & 37.58954 & 8.872671 & 11.62777 \\
\cline { 2 - 6 } & hGAPSO-SOS & 39.10687 & 39.21545 & 7.987178 & 7.789962 \\
\hline \multirow{5}{*}{ Glioma } & PSO & 32.27554 & 32.36587 & 38.50582 & 37.7132 \\
\cline { 2 - 6 } & BFOA & 32.84134 & 32.98745 & 33.80231 & 32.68401 \\
\cline { 2 - 6 } & hBFOA-PSO & 33.04264 & 33.25874 & 32.27129 & 30.70481 \\
\cline { 2 - 6 } & hGAPSO-SOS & 33.83699 & 33.90258 & 26.87704 & 26.47417 \\
\hline \multirow{5}{*}{ Astrocytoma } & PSO & 33.77889 & 33.81247 & 27.23901 & 27.02921 \\
\cline { 2 - 6 } & BFOA & 34.00706 & 34.12354 & 25.84487 & 25.16091 \\
\cline { 2 - 6 } & hBFOA-PSO & 34.35888 & 34.47895 & 23.83375 & 23.18384 \\
\cline { 2 - 6 } & hBFOA-PSO & 35.08465 & 35.45789 & 20.1658 & 18.5051 \\
\cline { 2 - 6 } & hGAPSO-SOS & 35.29112 & 35.65894 & 19.22952 & 17.66796 \\
\hline & PSO & 34.03859 & 34.25874 & 25.65791 & 24.38969 \\
\hline
\end{tabular}

Figure 5. Importance of WPSNR and VPSNR over PSNR
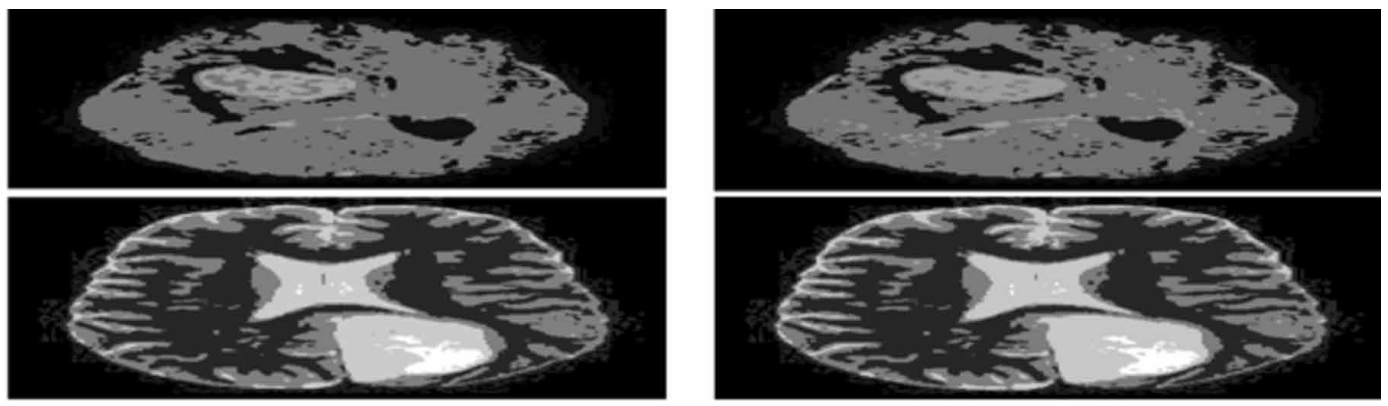

Astrocytoma Image
PSNR $=77.5538$
$\mathrm{MSE}=57.8177$
WPSNR $=-27.2145$
$\mathrm{FSIM}=0.62865$
Objective value $=11.0243$
SSIM $=0.6568$

Glioma Image

PSNR $=75.2909$

$\mathrm{MSE}=97.3549$

WPSNR $=-28.054$

FSIM $=0.680628$

Objective value $=12.547 \quad$ SSIM $=0.67674$
Astrocytoma Image

$$
\text { PSNR }=30.3473
$$

WPSNR $=-29.294$

$\mathrm{MSE}=30.3889$

$\mathrm{FSIM}=0.6865$

Objective value $=11.023 \quad$ SSIM $=0.6304$

Glioma Image

\begin{tabular}{c|c}
\hline PSNR $=28.2155$ & MSE $=49.64727$ \\
\hline WPSNR $=-28.0719$ & FSIM $=0.678498$ \\
\hline Objective value $=12.549$ & SSIM $=0.676002$ \\
\hline
\end{tabular}




\section{Weighted PSNR (WPSNR)}

The WPSNR incorporate human visual system into account while measuring the similarity between the original image and reconstructed image. WPSNR is HVS-based method and more accurate than PSNR. WPSNR uses the principle of redundancy of the human eye towards high frequency components in images. The human perception of vision is less sensitive to edges than smooth areas. WPSNR is nothing but PSNR weighted by the HVS parameter (Navas et al., 2011). The WPSNR in $\mathrm{dB}$ is expressed as

$$
W P S N R=10 * \log _{10}\left(\frac{(255)^{2}}{N V F \times M S E}\right)
$$

Where NVF is Noise Visibility Function defined as

$$
N V F=n o r m\left(\frac{1}{1+\delta_{b l o c k}^{2}}\right)
$$

Where ' $\delta$ ' block is the standard deviation of blocks of pixels having a specific size $(8 \times 8)$. In smooth regions, the value of NVF is near to zero and in the regions with edges, texture it is near to unity. The importance of WPSNR is explained in detail with the help of figure 5. From figure 5, it is observed that much difference in PSNR between left side and right side images but WPSNR value shows small difference. It shows that, PSNR failed to show right measure whereas WPSNR shows right measure. From Table 3 it is observed that, WPSNR is better with the 2-D histogram as compared to 1-D histogram.

Visual-PSNR (VPSNR): Another drawback with PSNR is that, sometimes it does not match with image expert Mean Opinion Scores (MOS). So visual PSNR is introduced by author Alexander (Tanchenko, 2014) and it is measured by considering visual MSE and contrast masking aspect of human visual system (Teo \& Heeger, 1994). They proved VPSNR measure outperforms existing SSIM, FSIM and WPSNR. The visual MSE is calculated by partitioning the input and output image into n blocks, $X$ and $Y$ are input and reconstructed images respectively, $N$ is image size and $K$ is size of partitioned image block then visual MSE is given as

$$
V M S E_{K}=\frac{M S E_{K}}{1+0.5 \sqrt{\sigma_{X}^{K} \sigma_{Y}^{K}}} K=1,2,3 \ldots \ldots \ldots . . N
$$

Where $M S E_{K}=\frac{1}{n} \sum_{i=1}^{n}\left(X_{i}^{K}-Y_{i}^{K}\right)^{2}$ and standard deviation of the block is calculated as follows

$$
\sigma_{X}^{K}=\sqrt{\frac{1}{n-1}} \sum_{i=1}^{n}\left(X_{i}^{K}-U_{i}^{K}\right)^{2} \text { and } U_{i}^{K}=\frac{1}{n} \sum_{i=1}^{n} X_{i}^{K}
$$


Table 3. Comparison of WPSNR \& VPSNR values for various algorithms

\begin{tabular}{|c|c|c|c|c|c|}
\hline \multirow[b]{2}{*}{ Images } & \multirow[b]{2}{*}{ Opt Tech } & \multicolumn{2}{|l|}{ WPSNR } & \multicolumn{2}{|l|}{ VPSNR } \\
\hline & & 1-D histogram & 2-D histogram & 1-D histogram & 2-D histogram \\
\hline \multirow{4}{*}{ Meningioma } & PSO & 16.0525 & 16.768 & 15.568 & 15.256 \\
\hline & BFOA & 18.1255 & 18.978 & 16.258 & 16.124 \\
\hline & hBFOA-PSO & 19.7084 & 20.024 & 17.478 & 17.102 \\
\hline & hGAPSO-SOS & 19.0003 & 20.147 & 19.985 & 19.258 \\
\hline \multirow{4}{*}{ Glioma } & PSO & 18.7953 & 18.987 & 14.457 & 16.909 \\
\hline & BFOA & 18.9130 & 18.978 & 14.698 & 18.024 \\
\hline & hBFOA-PSO & 19.1240 & 19.057 & 16.457 & 18.245 \\
\hline & hGAPSO-SOS & 19.2351 & 19.333 & 19.985 & 19.658 \\
\hline \multirow{4}{*}{ Coronary } & PSO & 18.1561 & 18.245 & 14.102 & 18.214 \\
\hline & BFOA & 19.9524 & 19.447 & 16.247 & 19.247 \\
\hline & hBFOA-PSO & 20.5253 & 20.727 & 16.367 & 22.124 \\
\hline & hGAPSO-SOS & 20.8112 & 20.963 & 18.247 & 22.247 \\
\hline \multirow{4}{*}{ Astrocytoma } & PSO & 18.6089 & 18.747 & 16.957 & 18.608 \\
\hline & BFOA & 19.5862 & 19.658 & 17.547 & 18.425 \\
\hline & hBFOA-PSO & 19.8229 & 19.919 & 18.654 & 19.962 \\
\hline & hGAPSO-SOS & 20.1235 & 20.207 & 19.102 & 20.753 \\
\hline
\end{tabular}

$V P S N R=10 * \log _{10}\left(\frac{\left(255^{2}\right)}{\overline{M S E}}\right)$

Where $\overline{M S E}=\frac{1}{N} \sum_{k=1}^{N} M S E_{K}$

From Table 3 it is observed that proposed method VPSNR is better with the 2-D histogram as compared to 1-D histogram. In addition to WPSNR and VPSNR, we measured Structural Similarity Index (SSIM), Feature Similarity Index (FSIM) are also measured which is shown in figure 5. From the above overall discussion the proposed method performance is better in accepted views.

\section{Qualitative Results}

Here we focused on visual clarity of reconstructed image which are obtained by thresholding the image in both 1-D histogram and proposed 2-D histogram by maximizing the Renyi entropy with proposed hybrid GAPSO-SOS and with PSO, BFOA and hBFOA-PSO algorithms. The reconstructed images and histogram obtained with 1-D histogram and proposed 2-D histogram with hybrid GAPSO-SOS algorithms at threshold level 5 with Renyi entropy are shown in Figure 6. As we know, at higher levels of threshold, the constructed image visual quality is much better. For efficiency measure of proposed Hybrid GAPSO-SOS, let us have a look at the visual quality of few reconstructed images with Renyi entropy: Coronary at 5 level thresholds as in Figure. 6b, Astrocytoma image at 5 level thresholds as in Fig. 6a from these figures GAPSO-SOS visual quality is better with 2-D histogram 
Figure 6. Reconstructed images with both hBFOA-PSO and hGAPSO-SOS a) Astrocytoma b) Coronary c) Glioma d) Meningioma

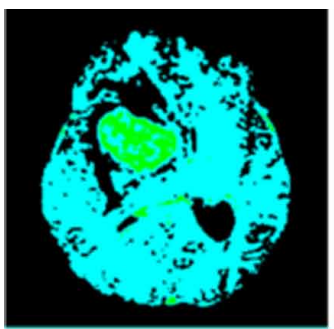

(a)
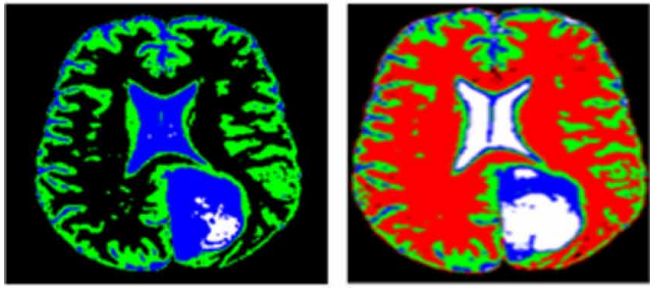

(c)

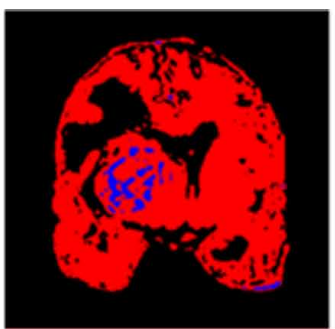

(b)
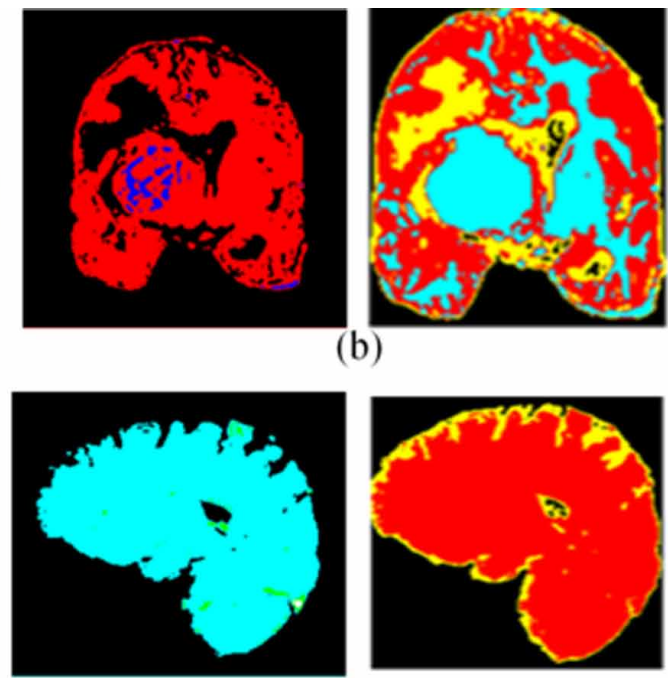

(d)

Table 4. Run time or computational time of various algorithms

\begin{tabular}{|c|c|c|c|c|c|}
\hline Images & Opt Tech & Th $=2$ & $\mathbf{T h}=\mathbf{3}$ & $\mathbf{T h}=4$ & $\mathbf{T h}=\mathbf{5}$ \\
\hline \multirow{4}{*}{ Meningioma } & PSO & 25.46385 & 13.69012 & 34.02886 & 33.71953 \\
\hline & BFOA & 10.52619 & 15.39352 & 11.84194 & 14.01381 \\
\hline & hBFOA-PSO & 46.19322 & 52.38832 & 55.73822 & 65.23239 \\
\hline & hGAPSO-SOS & 47.22294 & 56.49679 & 61.75288 & 70.42309 \\
\hline \multirow{4}{*}{ Glioma } & PSO & 10.01931 & 21.18469 & 16.58885 & 28.29965 \\
\hline & BFOA & 15.89806 & 12.32892 & 12.38941 & 11.85190 \\
\hline & hBFOA-PSO & 42.49393 & 45.29292 & 52.39332 & 60.39392 \\
\hline & hGAPSO-SOS & 46.39393 & 51.49382 & 57.39392 & 61.29292 \\
\hline \multirow{4}{*}{ Coronary } & PSO & 13.35572 & 18.43634 & 22.30330 & 15.81389 \\
\hline & BFOA & 10.11406 & 16.77978 & 11.74920 & 12.54278 \\
\hline & hBFOA-PSO & 45.39393 & 48.39922 & 55.33091 & 61.93030 \\
\hline & hGAPSO-SOS & 47.29292 & 51.29292 & 58.20022 & 62.32323 \\
\hline \multirow{4}{*}{ Astrocytoma } & PSO & 14.40273 & 24.59532 & 26.06671 & 31.13277 \\
\hline & BFOA & 22.75867 & 10.55672 & 10.12393 & 15.70930 \\
\hline & hBFOA-PSO & 42.54542 & 45.33493 & 56.39393 & 62.34342 \\
\hline & hGAPSO-SOS & 45.39393 & 48.33327 & 59.30302 & 65.39393 \\
\hline
\end{tabular}

as compare to 1-D histogram. Similarly, for Renyi entropy visual quality of proposed hBFOA-PSO is better with 2-D histogram. For example, Glioma image at 5 level thresholds as shown in 


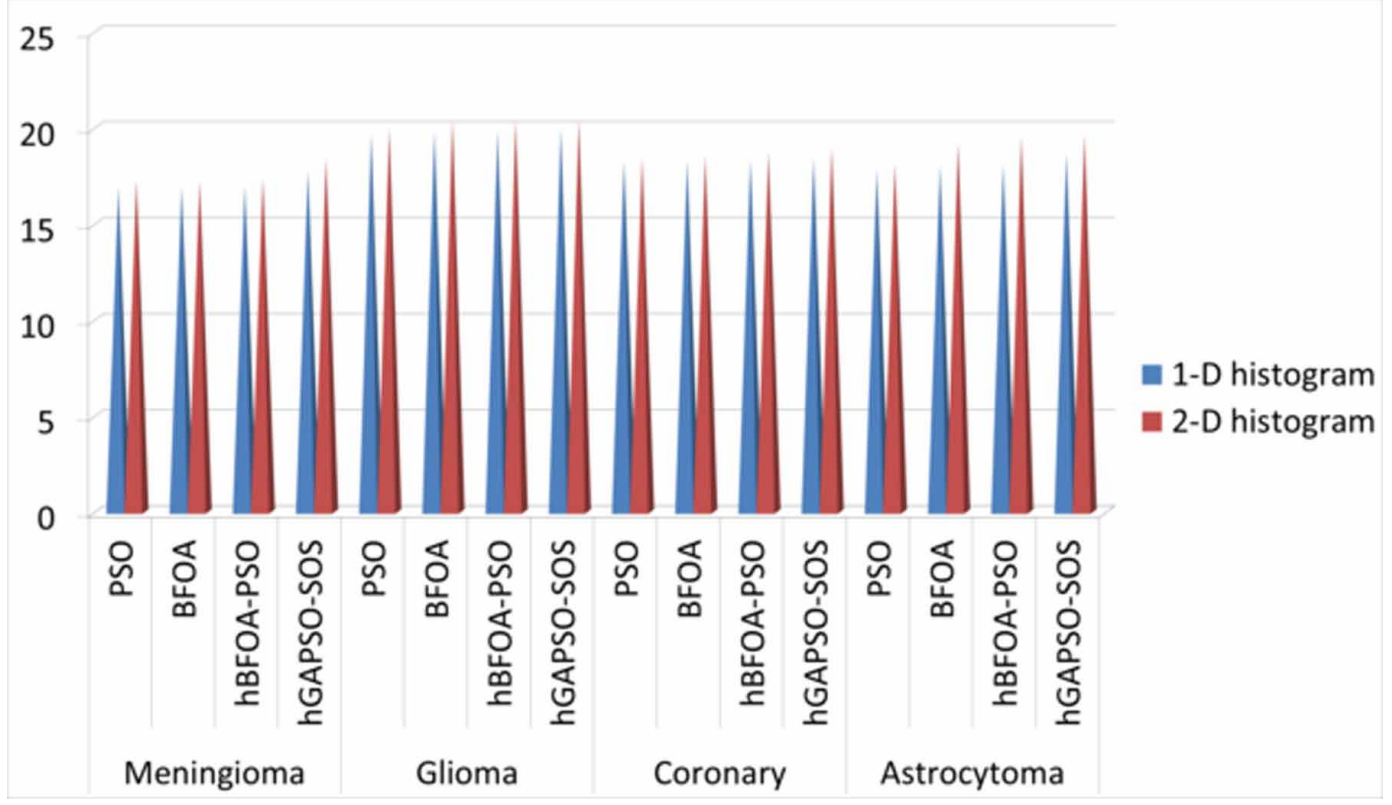

Fig. 6c and Meningioma image at 5- level thresholds as shown in Fig. 6d. Similarly, for all other images proposed algorithm is better compared to other earlier algorithms in visual quality. In Fig. 6d, the background in the Meningioma image is not visible with 1-D histogram at five level thresholds. But it is clearly visible with 2-D histogram. Moreover, as the numbers of thresholds are increased the image becomes clearly recognizable. Table 4 shows computation time of various algorithms. The computation time of proposed algorithm is more or less similar to hBFOA-PSO and it is less compared to other algorithms.

\section{CONCLUSION}

In this paper, hybrid combination of Genetic Algorithm, Particle Swarm Optimization and Symbiotic Organisms Search is used for 2-D histogram based multilevel image thresholding for image compression by maximizing the Renyi entropy for effective and efficient image thresholding. The proposed algorithm is successfully tested on standard test images to show the performance of the algorithm. The procured result of the hGAPSO-SOS is compared with other optimization algorithms such as PSO, BFOA and hBFOA-PSO with Renyi entropy. With these comparisons, it is observed that the proposed algorithm i.e., hGAPSO-SOS has a maximum fitness value among other algorithms. The proposed algorithm has higher PSNR, VPSNR, WPSNR values than PSO, BFOA and hBFOAPSO and more over improved quality of the reconstructed image is obtained. It is concluded that the proposed algorithm outperforms PSO, BFOA and hBFOA-PSO in all performance parameters.

Future Scope: The proposed work is further extended with the recent optimization techniques like Constriction Coefficient based Particle Swarm Optimization and Gravitational Search Algorithm (CPSOGSA) (Rather \& Bala, 2019), Grey Wolf Optimizer (GWO) (Mirjalili et al., 2014), and Sine Cosine Algorithm (SCA) (Mirjalili, 2016). 


\section{REFERENCES}

Ahmadi, K., Javaid, A. Y., \& Salari, E. (2015). An efficient compression scheme based on adaptive thresholding in wavelet domain using particle swarm optimization. Signal Processing: Image Communication, 32, 33-39. doi: 10.1016/j.image.2015.01.001

Bruylants, T., Munteanu, A., \& Schelkens, P. (2015). Wavelet based volumetric medical image compression. Signal Processing Image Communication, 31, 112-133. doi:10.1016/j.image.2014.12.007

Chan, T. F., \& Zhou, H. M. (2007). Total variation wavelet thresholding. Journal of Scientific Computing, 32(2), 315-341. doi:10.1007/s10915-007-9133-0

Cheng, M. Y., \& Prayogo, D. (2014). Symbiotic organisms search: A new metaheuristic optimization algorithm. Computers \& Structures, 139, 98-112. doi:10.1016/j.compstruc.2014.03.007

Chiranjeevi, K., \& Jena, U. (2017). Hybrid gravitational search and pattern search-based image thresholding by optimising Shannon and fuzzy entropy for image compression. International Journal of Image and Data Fusion, 8(3), 236-269. doi:10.1080/19479832.2017.1338760

Chiranjeevi, K., \& Jena, U. (2018). SAR image compression using adaptive differential evolution and pattern search based K-means vector quantization. Image Analysis \& Stereology, 37(1), 35-54. doi:10.5566/ias.1611

Chiranjeevi, K., Jena, U. R., \& Harika, A. (2017). Image Compression Using Shannon Entropy-Based Image Thresholding. In Computational Intelligence in Data Mining (pp. 101-110). Springer. doi:10.1007/978-98110-3874-7_10

De Luca, A., \& Termini, S. (1972). A definition of a nonprobabilistic entropy in the setting of fuzzy sets theory. Information and Control, 20(4), 301-312. doi:10.1016/S0019-9958(72)90199-4

Gregory, T. R. (2009). Understanding natural selection: Essential concepts and common misconceptions. Evolution (New York), 2(2), 156-175. doi:10.1007/s12052-009-0128-1

Grosan, C., \& Abraham, A. (2011). Intelligent systems. Springer. doi:10.1007/978-3-642-21004-4

Han, X., Quan, L., Xiong, X., Almeter, M., Xiang, J., \& Lan, Y. (2017). A novel data clustering algorithm based on modified gravitational search algorithm. Engineering Applications of Artificial Intelligence, 61, 1-7. doi:10.1016/j.engappai.2016.11.003

Haweel, R. T., El-Kilani, W. S., \& Ramadan, H. H. (2016). Fast approximate DCT with GPU implementation for image compression. Journal of Visual Communication and Image Representation, 40, 357-365. doi:10.1016/j. jvcir.2016.07.003

Hsu, W. Y. (2012). Improved watershed transform for tumor segmentation: Application to mammogram image compression. Expert Systems with Applications, 39(4), 3950-3955. doi:10.1016/j.eswa.2011.08.148

Jain, A. K., Duin, R. P. W., \& Mao, J. (2000). Statistical pattern recognition: A review. IEEE Transactions on Pattern Analysis and Machine Intelligence, 22(1), 4-37. doi:10.1109/34.824819

Karri, C. (2021). Secure robot face recognition in cloud environments. Multimedia Tools and Applications, 80(12), 18611-18626. doi:10.1007/s11042-020-10253-5

Karri, C., Cheikhrouhou, O., Harbaoui, A., Zaguia, A., \& Hamam, H. (2021). Privacy Preserving Face Recognition in Cloud Robotics: A Comparative Study. Applied Sciences (Basel, Switzerland), 11(14), 6522. doi:10.3390/ app11146522

Karri, C., \& Jena, U. (2016). Fast vector quantization using a Bat algorithm for image compression. Engineering Science and Technology, an International Journal, 19(2), 769-781. doi: 10.1016/j.jestch.2015.11.003

Kiruba, M., \& Sumathy, V. (2018). Register Pre-Allocation based Folded Discrete Tchebichef Transformation Technique for Image Compression. Integration (Amsterdam), 60, 13-24. doi:10.1016/j.vlsi.2017.07.003

Kumar, M., \& Vaish, A. (2017). An efficient encryption-then-compression technique for encrypted images using SVD. Digital Signal Processing, 60, 81-89. doi:10.1016/j.dsp.2016.08.011 
Mirjalili, S. (2016). SCA: A sine cosine algorithm for solving optimization problems. Knowledge-Based Systems, 96, 120-133. doi:10.1016/j.knosys.2015.12.022

Mirjalili, S., Mirjalili, S. M., \& Lewis, A. (2014). Grey wolf optimizer. Advances in Engineering Software, 69, 46-61. doi:10.1016/j.advengsoft.2013.12.007

Naidu, M. S. R., Kumar, P. R., \& Chiranjeevi, K. (2018). Shannon and fuzzy entropy based evolutionary image thresholding for image segmentation. Alexandria Engineering Journal, 57(3), 1643-1655. doi:10.1016/j. aej.2017.05.024

Navas, K. A., Gayathri, D. K., Athulya, M. S., \& Vasudev, A. (2011, September). MWPSNR: A new image fidelity metric. In 2011 IEEE Recent Advances in Intelligent Computational Systems (pp. 627-632). IEEE. doi:10.1109/RAICS.2011.6069386

Otsu, N. (1979). A Threshold Selection from Gray-level Histograms. Systems Man Cybernet. doi:10.1109/ TSMC.1979.4310076

RatherS. A.BalaP. S. (2019). Hybridization of constriction coefficient based particle swarm optimization and gravitational search algorithm for function optimization. 10.2139/ssrn.3576489

Rather, S. A., \& Bala, P. S. (2020). Swarm-based chaotic gravitational search algorithm for solving mechanical engineering design problems. World Journal of Engineering, 17(1), 97-114. doi:10.1108/WJE-09-2019-0254

Rather, S. A., \& Bala, P. S. (2020). A hybrid constriction coefficient-based particle swarm optimization and gravitational search algorithm for training multi-layer perceptron. International Journal of Intelligent Computing and Cybernetics, 13(2), 129-165. doi:10.1108/IJICC-09-2019-0105

Rényi, A. (1961, June). On measures of information and entropy. In Proceedings of the 4th Berkeley symposium on mathematics, statistics and probability (Vol. 1, No. 547). Academic Press.

Sajad Ahmad Rather. (n.d.a). https://github.com/SajadAHMAD1

Sajad Ahmad Rather. (n.d.b). https://in.mathworks.com/matlabcentral/profile/authors/6240015-sajad-ahmadrather

Salleh, M. F. M., \& Soraghan, J. (2007). A new multistage lattice vector quantization with adaptive subband thresholding for image compression. EURASIP Journal on Advances in Signal Processing, 2007(1), 1-11. doi:10.1155/2007/92928

Sarkar, S., \& Das, S. (2013). Multilevel image thresholding based on 2D histogram and maximum Tsallis entropy-A differential evolution approach. IEEE Transactions on Image Processing, 22(12), $4788-4797$. doi:10.1109/TIP.2013.2277832 PMID:23955760

Seyedali Mirjalili. (n.d.). https://www.mathworks.com/matlabcentral/profile/authors/2943818

Sezgin, M., \& Sankur, B. (2004). Survey over image thresholding techniques and quantitative performance evaluation. Journal of Electronic Imaging, 13(1), 146-166. doi:10.1117/1.1631315

Sidhik, S. (2015). Comparative study of Birge-Massart strategy and unimodal thresholding for image compression using wavelet transform. Optik (Stuttgart), 126(24), 5952-5955. doi:10.1016/j.ijleo.2015.08.127

Skodras, A., Christopoulos, C., \& Ebrahimi, T. (2001). The jpeg 2000 still image compression standard. IEEE Signal Processing Magazine, 18(5), 36-58. doi:10.1109/79.952804

Sunder, R. S., Eswaran, C., \& Sriraam, N. (2006). Medical image compression using 3-D Hartley transform. Computers in Biology and Medicine, 36(9), 958-973. doi:10.1016/j.compbiomed.2005.04.005 PMID:16026779

Tanchenko, A. (2014). Visual-PSNR measure of image quality. Journal of Visual Communication and Image Representation, 25(5), 874-878. doi:10.1016/j.jvcir.2014.01.008

Teo, P. C., \& Heeger, D. J. (1994, November). Perceptual image distortion. In Proceedings of 1st International Conference on Image Processing (Vol. 2, pp. 982-986). IEEE. doi:10.1109/ICIP.1994.413502

Venugopal, D., Mohan, S., \& Raja, S. (2016). An efficient block based lossless compression of medical images. Optik (Stuttgart), 127(2), 754-758. doi:10.1016/j.ijleo.2015.10.154 
Yang, X.-S. (2010). Nature-Inspired Metaheuristic Algorithms (2nd ed.). Luniver Press.

Zhang, M., \& Tong, X. (2017). Joint image encryption and compression scheme based on IWT and SPIHT. Optics and Lasers in Engineering, 90, 254-274. doi:10.1016/j.optlaseng.2016.10.025

Zhang, M., \& Tong, X. (2017). Joint image encryption and compression scheme based on IWT and SPIHT. Optics and Lasers in Engineering, 90, 254-274. doi:10.1016/j.optlaseng.2016.10.025

Karri Chiranjeevi received a B.Tech. in 2003 from JNTU Hyderabad, M.tech. in 2003 from JNTU Kaikinada and PhD from Veer Surendra Sai University of Technology, India. He is working under project sponsored by FCT at Computational Clinical Imaging Group (CCIG), Champalimaud Foundation Centre for the Unknown, Portugal. He was a post doctoral researcher in Universidade da Beira Interior, Portugal from 2019 to 2020. He is Associate Professor in the department of Electronics and Communication Engineering, GMR institute of Technology, India. His research interests include Machine learning, Cloud Robotics and Computer vision. He is editor and reviewer of various international journals. He is having an experience of teaching 14 years and research experience of 5 years also having 40 national and international journals and conferences.

G. Ramesh Babu is currently working as Professor in the department of Electronics and Communication Engineering, Raghu Engineering College (Autonomous), Visakhapatnam, Andhra Pradesh, India. He received his Bachelor of Technology (B. Tech) in Electronics and Communications Engineering from Nagarjuna University, Andhra Pradesh (1994-1998), and obtained his Master of Engineering (M.E) from PSG College Of Technology , Coimbatore, Tamilnadu (2001) and Doctor of Philosophy PhD in Electronics and Communication Engineering from Osmania University, Hyderabad, India (2014). He has over 16 Years and 7 months of teaching and industry experience. Prof. Ramesh Babu has served as Principal, in Sri Sivani Institute of Technology Srikakulam, AP, India during July 2015 - Feb 2018 and also Vice-principal, in Sri Sivani College of Engineering during Jan 2014-June 2015 and served as $H O D$ in various colleges. He has taught several undergraduate and graduate courses in Signal Processing \&Communication Engineering area and supervised nearly $20 \mathrm{UG}$ and 15 PG student projects in the area of Signal processing and communication systems. He published more than 20 publications which include journal papers, conference papers. His research interests are in the areas of signal processing, non-stationary signal processing, speech processing, Image processing. He is a Life Member of Institution of Engineers (IE), Indian Society for Technical Education (ISTE).

P. M. K. Prasad received PhD in Electronics and Communication Engineering from Andhra University, Visakhapatnam and ME in Systems and Signal Processing from Osmania University Hyderabad. He has 25 years of experience in teaching. He is working as an Associate Professor, Department of ECE in GVP College of Engineering for Women Visakhapatnam, India since 2017. He has published more than 50 papers in national/international journals/ conferences. His research interests are in Signal Processing, Communications, and Image Processing.

M. S. R. Naidu received B. Tech. in 2002 from JNTU Hyderabad, M. Tech. in 2010 from JNTU Kaikinada and PhD from Andhra University of Technology, India. He is Associate Professor in the department of Electronics and Communication Engineering, Aditya Institute of Technology and Management Tekkali, India. His research interests include optimization and Computer vision. He is having an experience of teaching 17 years having 13 national and international journals and conferences. 